\title{
Examining the Cycling Behaviour of Li-Ion Batteries Using Ultrasonic Time-of-Flight Measurements
}

\author{
James B. Robinson ${ }^{1,2 *}$, Martin Pham ${ }^{1,2,3}$, Matt D.R. Kok ${ }^{1,2,3}$, Thomas M.M. Heenan ${ }^{1,2,3}$, Dan J. \\ L. Brett ${ }^{1,2,3}$ and Paul R. Shearing ${ }^{1,2,3}$ \\ ${ }^{1}$ Electrochemical Innovation Lab, Department of Chemical Engineering, UCL, London, WC1E 7JE, UK \\ ${ }^{2}$ Electrochemical Diagnostics \& Forensics Lab, Department of Chemical Engineering, UCL, London, WC1E 7JE, \\ $\mathrm{UK}$ \\ ${ }^{3}$ The Faraday Institution, Quad One, Harwell Science and Innovation Campus, Didcot, OX11 ORA, UK \\ * Author to whom correspondence should be addressed \\ Web: https://www.ucl.ac.uk/electrochemical-innovation-lab/; https://www.ucl.ac.uk/electrochemical-innovation- \\ lab/innovation-and-outreach/forensic-and-diagnostic-lab \\ Email: j.b.robinson@ucl.ac.uk; d.brett@ucl.ac.uk; p.shearing@ucl.ac.uk
}

\begin{abstract}
Diagnostic systems for Li-ion batteries have become increasingly important due to the larger size, and cost of the batteries being deployed in increasingly demanding applications, including electric vehicles. Here, ultrasound acoustic time-of-flight (ToF) analysis is conducted under a range of operating conditions. Measurements are performed on a commercial pouch cell during varying discharge rates to identify a range of effects that influence the acoustic ToF measurements. The cell was examined using X-ray computed tomography to ensure no significant defects were present and to confirm the layered structure in the region being investigated, validating the signal pattern observed. Analyses of the acoustic signals obtained suggest that stresses are generated in the electrodes during both the charge and discharge of the cell with the magnitude of Young's modulus for the component materials being both a function of the state-of-charge and applied current. Characteristic responses for both electrodes during the charge/discharge cycle highlight the potential application of the technique as a real-time diagnostic tool.
\end{abstract}

\section{Keywords}

Ultrasound acoustics; Li-ion battery; battery diagnostics; electrochemical stiffness; X-ray computed tomography. 


\section{Introduction}

Lithium-ion (Li-ion) batteries are ubiquitous in personal computing, in addition to the everincreasing electric vehicle (EV) market [1-3]. The Li-ion battery benefits from comparatively high power and energy densities and relatively long cycle life; however, there is a significant impetus to improve these factors, particularly for demanding EV applications. The development of diagnostic tools and sophisticated battery management strategies has seen improvements in the performance of cells by limiting their operation to defined limits. This balances the performance of the cell with the mitigation of rapid degradation, which occurs at the extremes of operational voltages [4-8].

Diagnostic techniques have used an array of metrics, including electrochemical and physical measurements, to ascertain safe operating conditions [9]. State-of-charge (SoC) estimation in EVs can be performed in-operando by measuring several properties of a battery, including the voltage, discharge current and impedance [10], which is then analysed in the battery management system (BMS) of the vehicle [11-15]. While these techniques are relatively robust, more direct methods such as Coulomb (charge) counting are susceptible to accumulated errors due to external factors, including temperature and the temporal resolution of the measurements [16]. The SoC measurements provide limited insight into the physical condition of the electrodes of the battery, which may indicate potential degradation and failure of the cells.

The physical measurement of batteries has been widely described using X-ray computed tomography (CT) methods [17]. While there is scope to use X-ray CT in cell manufacturing [18], it is not a viable solution on vehicles; however, it can provide a priori insight into the internal architecture of the cell, which can be very useful to inform the results obtained with other techniques. One of the more common operando techniques in electric vehicle applications involves the analysis of the thermal performance of batteries [19], which can provide indications of faults [20] or non-ideal operation of cells [21], and benefit from simple integration into EV battery packs. While these techniques can provide insights into the stateof-health ( $\mathrm{SoH})$ of cells [22] it is not possible to determine the SoC of cells in this manner.

Ultrasound acoustic measurements have the potential to determine both the $\mathrm{SoH}$ and $\mathrm{SoC}$ of batteries in a compact, cost-effective manner [23]. Due to the regular, layered internal structure of Li-ion batteries, acoustic ToF analysis has the potential to directly identify changes in the 
electrode structure. These changes can include operational alterations in the dimensions of the electrodes due to variations in lithiation state or failures (including delamination and cracking). Hsieh et al. highlighted variations in the acoustic ToF signal in Li-ion batteries due to cell cycling at very low C-rates (C/20) [24]; this work was followed by Davies et al. who developed a model to describe the performance of the cell [23]. These studies highlighted the potential of acoustic ToF measurements as a tool to track the degradation of cells due to low-rate cycling. Bhadra et al. examined $\mathrm{Zn} / \mathrm{MnO}_{2}$ cells and quantified the variations in the acoustic ToF signal due to the oxidation of the anode associated with discharging of the primary cells [25]. This work took advantage of significant changes in the properties of component materials in primary cells, correlating these changes to the SoC of the cells.

Previous work conducted by Robinson et al. highlighted interactions between the electrodes and architectural components of the cell using acoustic ToF measurements to highlight the anchoring effect of current collectors in commercial cells [26]. Ultrasound measurements conducted using guided waves were employed in Li-ion cells by Ladpli et al. to monitor the effect of cycling on cells [27] detailing a reduction in the acoustic ToF with increased cycling. These findings indicated an increase in acoustic wave propagation velocity in the cell over 200 cycles, highlighting the potential of acoustic techniques to provide information pertaining to the state-of-health of the cell over an extended period.

Here, temporally resolved pulse-receive acoustic ToF measurements are performed on Li-ion batteries to investigate the effects of both charging and discharging. By varying the C-rate applied to the cells during the cycling process, it is possible to identify changes in the cell's behaviour, demonstrating the potential implementation of acoustic ToF measurements in operando applications that require high-rate discharging, including EVs. The internal structure of the battery, obtained using X-ray CT is used to describe the received waveforms and provide further insight into the cell's behaviour.

\section{Experimental}

Ultrasound measurements were performed on commercial $210 \mathrm{mAh}$ batteries composed of a $\mathrm{LiCoO}_{2}$ (LCO) cathode with a graphite anode (PL-651628-2C, AA Portable Power Corp., Richmond, CA, USA) with a maximum recommended discharge of $2 \mathrm{C}$. Fresh cells were cycled from their initial SoC of ca. $3.8 \mathrm{~V}(20 \% \mathrm{SoC})$ to $4.2 \mathrm{~V}(100 \% \mathrm{SoC})$ at $210 \mathrm{~mA}$ (1C) using a 
Gamry Interface 1010E potentiostat (Gamry Instruments, Pennsylvania, USA). This upper voltage was held constant until the current dropped below $2.1 \mathrm{~mA}$. The cells were then discharged using a $\mathrm{C}$-rates ranging from $1-4 \mathrm{C}$ to a lower voltage of $2.7 \mathrm{~V}$, as shown in Figure 1(a).

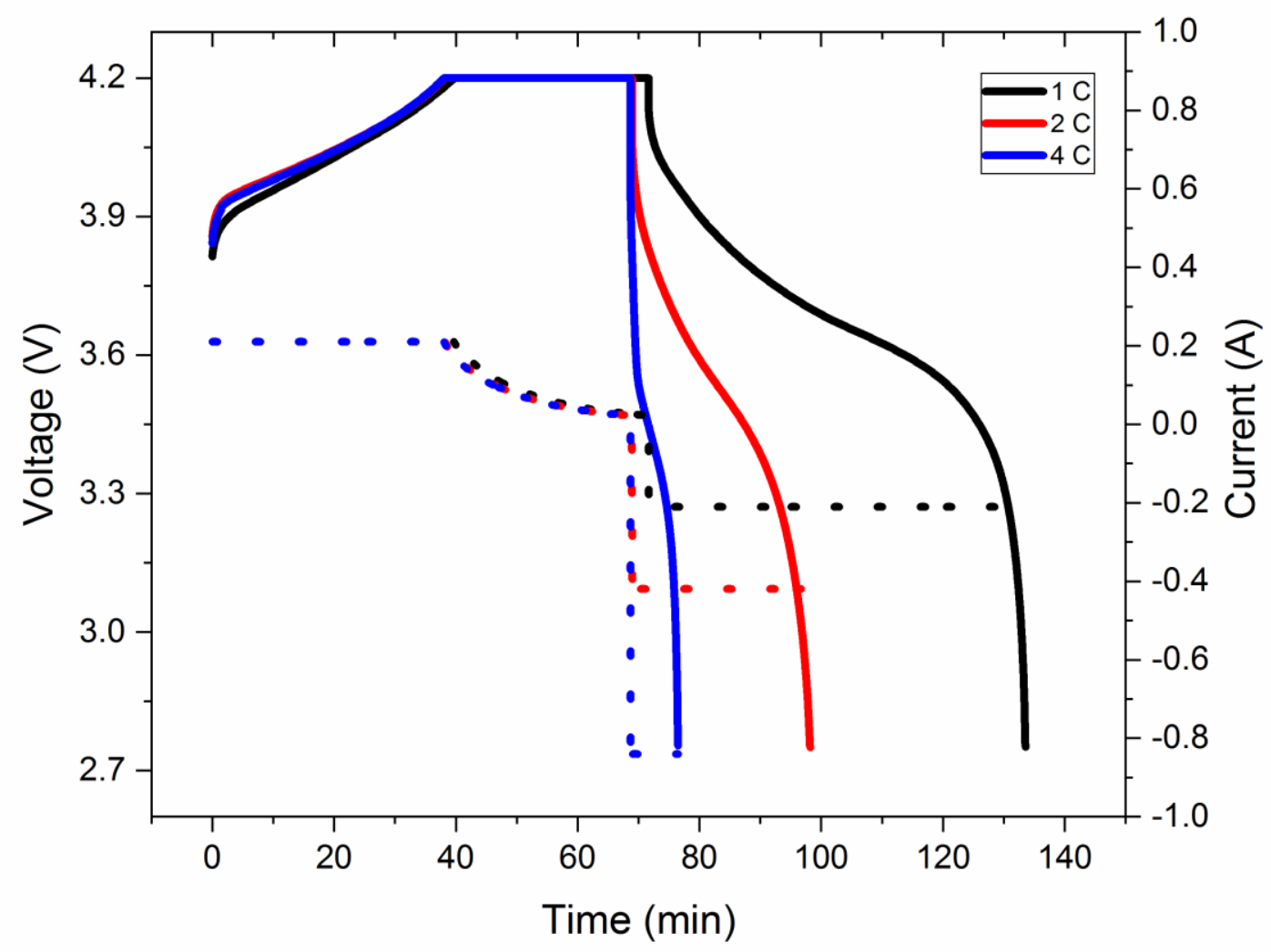

Figure 1 Voltage (shown in solid lines) and current (dashed lines) profiles of the cells cycled from an initial $3.8 \mathrm{~V}$ to $4.2 \mathrm{~V}$ followed by discharges at 1,2 and $4 \mathrm{C}$ respectively.

The acoustic ToF measurements were obtained simultaneously using an Olympus Epoch 650 ultrasonic flaw detector (Olympus Corp., Japan) with a $6 \mathrm{~mm}$ diameter piezoelectric transducer that operated at a frequency of $2.25 \mathrm{MHz}$ (M110-RM, Olympus Corp., Japan) used for all measurements. A thin layer of ultrasonic gel (VWR International, USA) was used to facilitate the ultrasonic measurements with consistent contact between the transducer and battery maintained by placing a $200 \mathrm{~g}$ weight on top of the transducer prior to all measurements, which were typically obtained using an acoustic amplitude of $60 \mathrm{~dB}$. The acoustic signal was obtained using an opensource Python code, described previously [24, 28], which connected to the flaw detector through an RS232 connection. This limited the acoustic ToF resolution to 495 discrete measurements, to 
maximise the information obtained, the total time window analysed was limited to $10 \mu$ s, providing a resolution of $20.2 \mathrm{~ns}$.

Thermal imaging of the battery was performed to examine whether the heating associated with charging/discharging resulted in significant changes to the properties (including density, Young's modulus, etc.) of the cells' component materials. This was achieved using an infrared camera during the same 1C charge-discharge cycle described previously. The infrared imaging was conducted using a FLIR X6903sc with a $25 \mathrm{~mm} \mathrm{~F} / 2.5$ lens giving an approximate pixel resolution of $65 \mu \mathrm{m}$ with images recorded at $1 \mathrm{~Hz}$. The images were subsequently analysed using FLIR ResearchIR with the average temperature of the cell, obtained from approximately 68,000 points, extracted for the full duration of the discharge. To examine the thermal sensitivity of the acoustic ToF technique, measurements were performed at the extremes of temperatures observed during the charging/discharging cycle. A similar battery to those used during the cycling was placed in a climate-controlled chamber at its initial open circuit voltage and allowed to equilibrate for 30 mins before five acoustic measurements were recorded to allow for improved accuracy. No charging or discharging of the battery was performed while it was in the climate-controlled chamber and the battery voltage before and after the tests was measured to ensure no damage to the cell was caused; in all cases, the potential was observed to be $3.8 \mathrm{~V}$.

The internal structure of the full battery was identified using X-ray CT performed using a Nikon XT-225 instrument (Nikon Metrology, Tring, UK). These scans were obtained at an accelerating voltage of $180 \mathrm{kV}_{\mathrm{p}}$ and an incident beam power of $18.5 \mathrm{~W}$ using a tungsten target and a $1 \mathrm{~mm} \mathrm{Cu}$ filter to reduce the effect of low energy X-rays and improve the quality of the radiographs. In total, 3176 projections were obtained for the scan with the geometric magnification of the system resulting in a pixel size of approximately $24.5 \mu \mathrm{m}$, which enabled the full visualisation of the electrode layers and current collection tabs within the cell. Reconstruction of the radiographic images was performed using Nikon CT Agent with visualisation achieved using Avizo Fire 9.4 (FEI, France). Radiographic videos of the cells' electrodes during cycling were obtained concurrently to the acoustic analysis using the Nikon XT-225 (in radiography mode) under the same conditions and are provided as supplementary information.

High-resolution tomographic imaging of the battery was performed using a Zeiss Xradia Versa 520 (Zeiss, Carl Zeiss., CA, U.S.A.) using an accelerating voltage of $80 \mathrm{kV}_{\mathrm{p}}$ with a tungsten source, yielding an isotropic pixel resolution of $7.7 \mu \mathrm{m}$. The acquired datasets were reconstructed using 
Zeiss' Reconstructor Scout-and-Scan software with visualisation and analysis of the electrode structure performed using Avizo Fire 9.4.

\section{Results and Discussion}

To gain a full understanding of the acoustic ToF results, it is necessary to understand the internal structures of the battery. As such, X-ray CT was performed to provide a description of the architecture of the cell, as shown in Figure 2. The three-dimensional rendering (Figure 2a) shows no large current collecting tab through the centre of the cell which can affect the acoustic ToF measurements [26]. To achieve the most consistent measurement, it was chosen to perform the acoustic ToF analysis towards the bottom of the cell, away from the cell terminals, which have been shown to have the potential to cause variations changes in acoustic ToF signal [26]. This location reduced the possibility of the acoustic ToF signal being distorted due to the current collecting tabs and manufacturing misalignments in the cell. The orthogonal slices shown in Figure 2(b) highlight the layered structure of the cell, which occurs throughout the majority of the battery. It can be seen that the battery is composed of regularly spaced, double layer anode and cathode layers (isolated from each other by a thin polymer separator). By understanding the architecture of the cell, it is possible to elucidate changes in the structure. These changes arise due to the electrochemical operation of the device and provide an opportunity for in situ diagnostics to be performed using acoustic ToF measurements. It can also be seen that there are no significant defects or delamination in the fresh cell, which may result in an anomalous acoustic reading, indicative of a cell failure. 

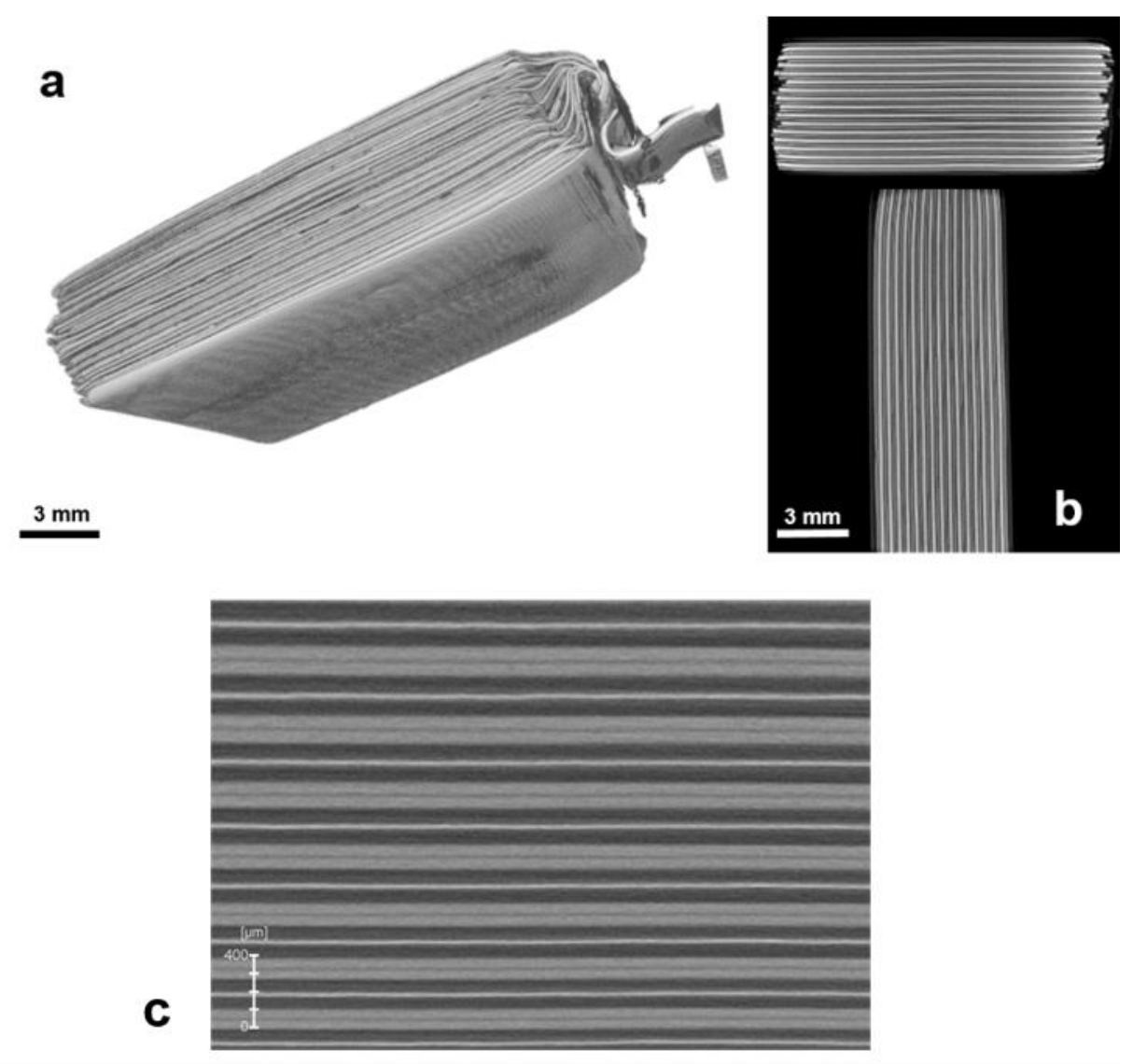

Figure 2 (a) Three-dimensional volume rendering of the $210 \mathrm{mAh}$ battery (with external casing digitally removed) examined highlighting the layered electrode structure and (b) internal configuration of the layered battery structure showing no defects in two orthogonal directions. A high-resolution internal tomography of the cell (c) shows the layered structure in greater detail, including the current collector separating the double layer electrode assembly.

The interpretation of the obtained acoustic signals is informed by the geometry of the cell. Due to the regularity of the structure shown in Figure 2(b), the acoustic ToF response (shown in Figure 3) can be expected to consist of a series of peaks which occur due to reflections from interfaces at which density changes occur. The resolution of the recording, discussed previously, will determine the number of peaks obtained. Examination of the high-resolution image in Figure 2(c) indicates that the anode and cathode double layers (including current collectors) are approximately $210 \mu \mathrm{m}$ and $150 \mu \mathrm{m}$ respectively. The current collectors were measured to be $20 \mu \mathrm{m}$ within the anode and $15 \mu \mathrm{m}$ in the cathode. Reported velocities in Li-ion battery materials are of the order 2000-3000 $\mu \mathrm{m} \mu \mathrm{s}^{-1}$ [23] implying that the time resolution used in this work enables depth probing at the ca. 40-60 $\mu \mathrm{m}$ resolution. This resolution results in an expectation of 4-6 individual spectra to form each peak, which is observed and discussed later in Figure 7. As such, the peaks observed in Figure 3 can be interpreted to be caused by material 
interfaces which result in a change in density, in this case most likely to be the electrode/current collector interface. This location is most likely due to the comparatively large difference between the ultrasound velocities (and results shown later in Figure 7). A characteristic exponential decay in the signal can also be observed in Figure 3. This drop in signal amplitude occurs due to the increased depth at which these peaks occur; and can be explained by the lower energy of the incident wave, which is reflected by the material interfaces at these depths.

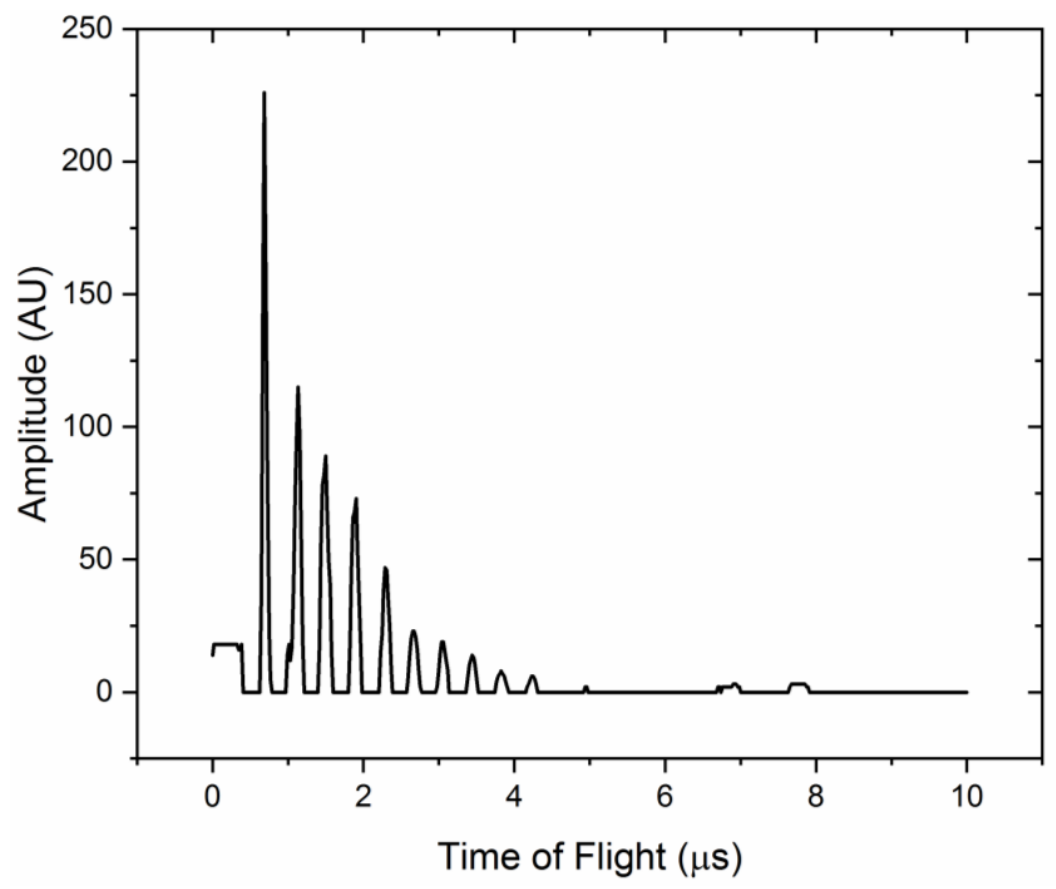

Figure 3 Example of a rectified acoustic signal obtained from the $210 \mathrm{mAh}$ cell taken at $20 \%$ SOC prior to the charging of the cell.

The interpretation of acoustic ToF measurements is complicated by various phenomena, which behave in differing manners within the cell during operations. While there is an expansion and contraction of the anode and the $\mathrm{LiCoO}_{2}$ cathode due to the electrochemical processes [29, 30] there is also a change in the physical properties of the component materials that results in a change to the ultrasound velocity through the materials. The changes in both the physical size and ultrasonic velocity associated with charging are principally governed by the change in density of the electrodes, a physically challenging parameter to measure in operando. The variation in the material condition also results in changes to parameters including the bulk $(K)$ and shear $(G)$ modulus, both related to the Young's modulus $(E)$ and Poisson's ratio $(v)$, which further affects the propagation velocity of waves through the material. The variation in material velocity can be described using a series of equations (shown below in Equations 1-3) which 
relate the velocity of wave propagation to the density $(\rho)$, Young's modulus and Poisson's ratio [23].

$$
\begin{aligned}
& C=\sqrt{\frac{K+\frac{4}{3} G}{\rho}} \\
& K=\frac{E}{3(1-2 v)} \\
& G=\frac{E}{2(1+v)}
\end{aligned}
$$

Previously, calculations performed based on the assumption of a constant Young's modulus [26] show the velocity of sound waves in LCO and graphite vary in opposite magnitudes during charging, i.e. the velocity in graphite increases with the velocity in LCO decreasing during charge [26]. This effect may be further complicated due to reported non-linearities in the Young's modulus throughout the possible lithiation states which results in the velocity initially reducing before rising above the initial value during the charging cycle for the active materials of both electrodes [23]. Voltage-dependent variations in the Young's modulus will also affect the acoustic impedance $(Z)$ of the component materials which will effect the acoustic amplitude of the signal as previously discussed [24, 26]. The acoustic impedance is also dependent upon the density $(\rho)$ as shown in Equation 4.

$$
Z=\sqrt{\rho E}
$$

Currently, absolute values of the velocities through battery component materials are not well described both due to the relative nascence of this field and the complex microstructure of the materials which form the electrodes and may have spatially variant porosity and density [31]. Further complications arise from the local heterogeneity of current distribution which may result in a large number of velocities across a planar electrode [32]. Improved knowledge of this behaviour is required to obtain absolute values to describe the physical change in the location of the electrode interfaces. However, comparative results, examining the cell over the same range of conditions (in this case a charge-discharge cycle between $2.7-4.2 \mathrm{~V}$ ), can provide insight into structural changes, the state-of-health and charge of the cell.

The acoustic ToF response for a charge-discharge cycle is shown in Figure 4. This response is a temporally resolved collection of individual signals collected over the full duration of the cycling experiments. The signals shown in Figure 4(a) are indicative of the responses, which 
comprise Figure 4(b); with the changes in the signal response observed in Figure 4(b) a direct result of changes in the location and/or amplitude of the peaks which form the acoustic ToF signal.

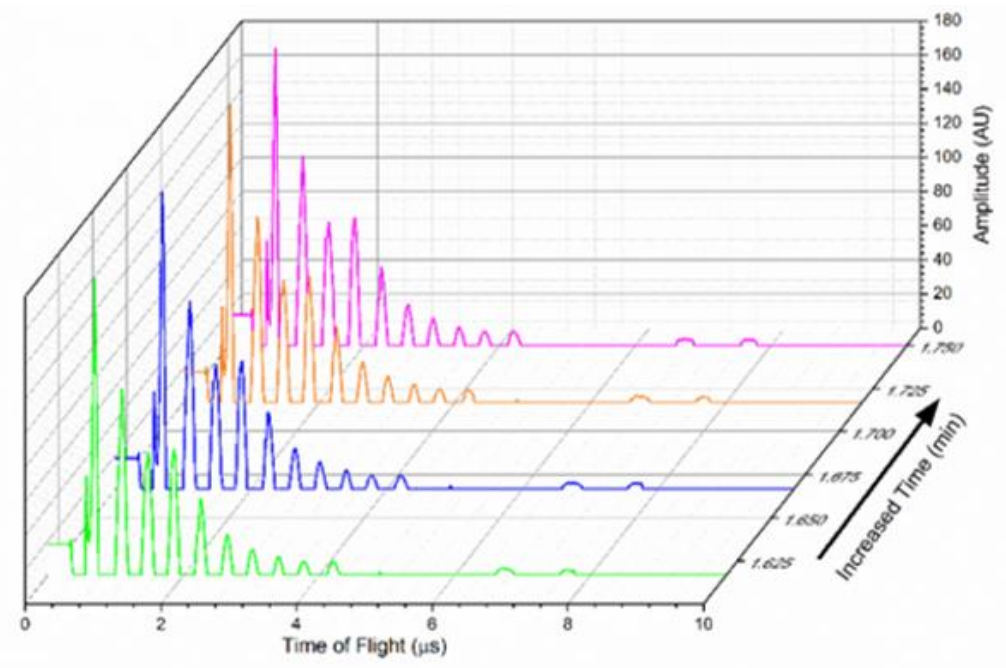

(a)
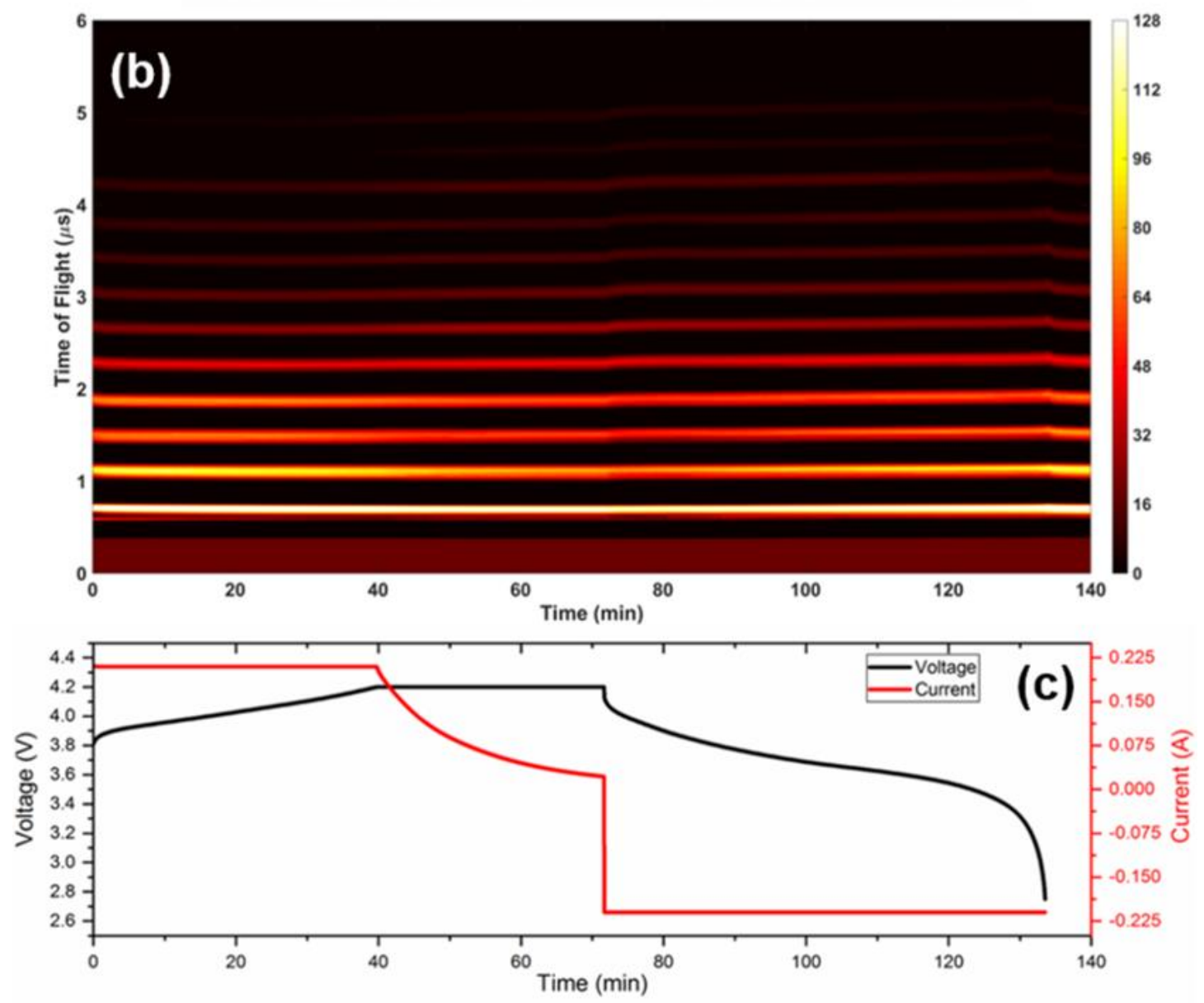

Figure 4 The acoustic signal responses (a) from a series of individual spectra similar to those shown in Figure 3 from the $210 \mathrm{mAh}$ cell. The responses for the full duration of a $1 \mathrm{C}$ charge/discharge cycle are 
shown in (b) with the electrochemical cycle information shown in (c). The acoustic ToF spectra [in (b)] shows the acoustic amplitude (measured in $\mathrm{AU}$ ) and a period during which the cell is allowed to maintain an open circuit potential.

Figure 4 highlights a number of the characteristic trends observed in the temporally resolved acoustic ToF signals obtained from a battery undergoing a $1 \mathrm{C}$ charge-discharge cycle. Small decreases in the acoustic ToF of the peaks are seen during the initial constant current (CC) charge from $0-72 \mathrm{~min}$. The acoustic ToF can be observed to uniformly increase due to the discharging of the cell until the discharging stops, this is consistent with previously reported results [26]. Interestingly, after the charging stops, there is a significant decrease in the acoustic ToF response.

To assess the potential impact of heating within the cell which may result in thermal expansion of the electrodes and changes to the density and physical parameters (including Young's and bulk moduli), tests were conducted to ascertain the maximum heating observed within the battery. Following this, acoustic measurements taken at the extremes of the temperature range the battery was exposed to in a climate controlled environment. The total heating at the surface of the cell was observed to be very small $\left(1-2{ }^{\circ} \mathrm{C}\right)$ within the majority of the charge cycle with a maximum temperature rise of ca. $5^{\circ} \mathrm{C}$ as the cell voltage dropped towards $2.7 \mathrm{~V}$. This comparatively small temperature rise is to be expected due to the small cell capacity ( $210 \mathrm{mAh})$ and relatively thin form factor (6.5 mm thickness). The results of the acoustic tests are shown in Figure 5, which show the similarity in the signals at both $25^{\circ} \mathrm{C}$ and $30^{\circ} \mathrm{C}$. Small changes in the acoustic intensity are observed which may be due to slight variations in the Young's modulus; however, it can be seen that no significant change in the ToF is caused by the increase in cell temperature. 

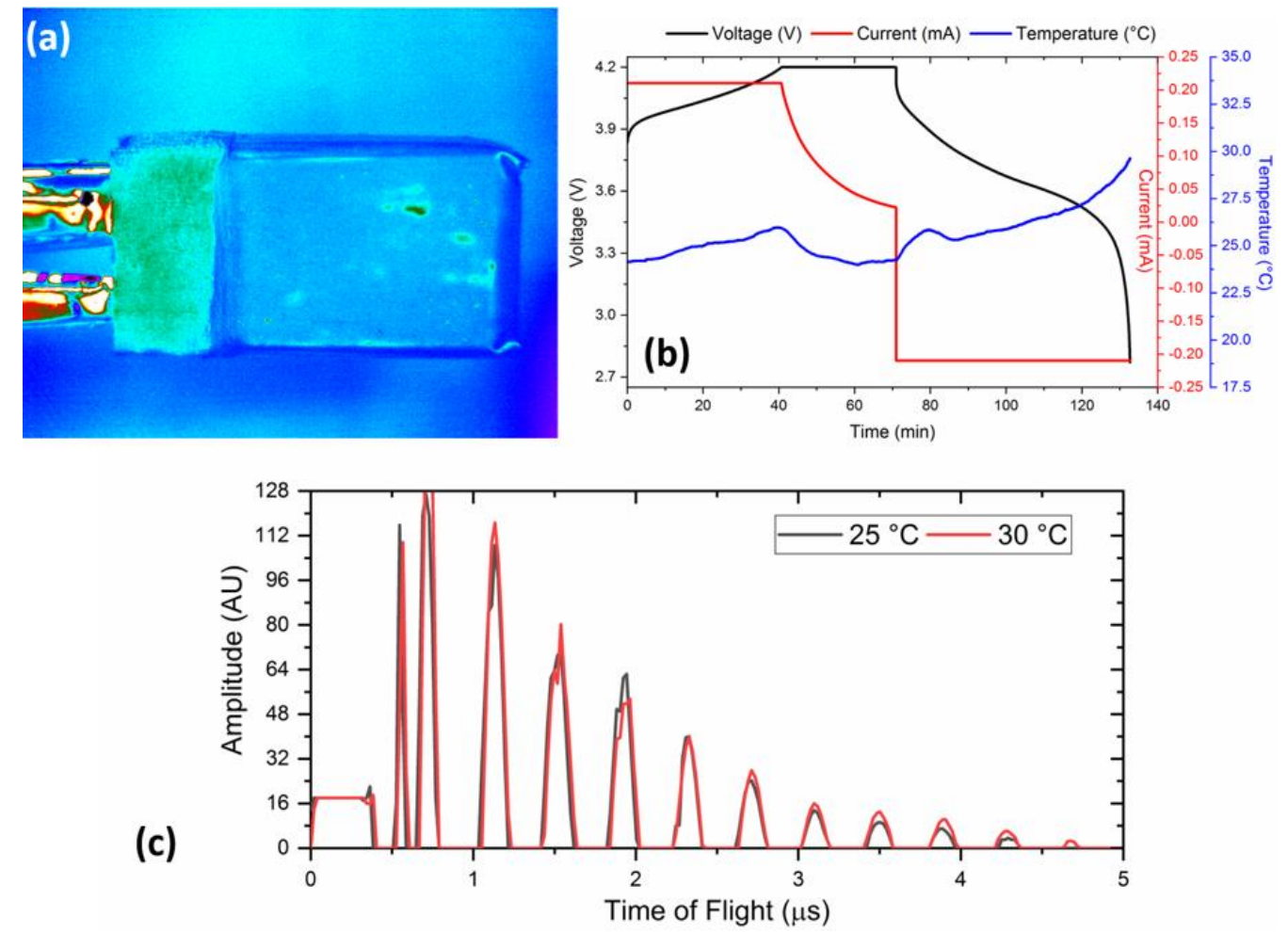

Figure 5 (a) Infrared image of a $210 \mathrm{mAh}$ battery used to examine the maximum temperature range with the thermal profile shown during the $1 \mathrm{C}$ charge/discharge cycle shown in (b) highlighting a maximum temperature of $30{ }^{\circ} \mathrm{C}$. Also shown in (c) is a comparison of the acoustic signals obtained at $25{ }^{\circ} \mathrm{C}$ and $30{ }^{\circ} \mathrm{C}$ showing no significant peak shifts over the range of temperatures observed for a $1 \mathrm{C}$ charge/discharge cycle.

The lack of change in the acoustic ToF over the temperature range shown above is to be expected due to the low change in absolute temperature observed during the discharge. While it is difficult to obtain exact values for battery materials, it has been shown that the thermal expansion coefficients of isotropic graphite and LCO are of the order of $10^{-5}-10^{-6}\left(\mu \mathrm{m} \mu \mathrm{m}^{-1}\right)$ ${ }^{\circ} \mathrm{C}^{-1}$, although in the case of LCO this is reported from $50{ }^{\circ} \mathrm{C}$ [33-35]. This places the thermal expansion coefficient of the electrode materials in the same order of magnitude as the metallic current collector components [36,37], which limits the potential to discretise these layers based on this effect. There is, however, significant scope for fundamental studies examining the effect of temperature on acoustic signals, and in turn, materials properties, for battery materials over the temperature range, compositional mixtures and lithiation states expected. Once more, the small temperature variation experienced by the cell is anticipated to result in very small changes to the density and Young's modulus of the component materials, with graphite, for example, reducing in density by less than $0.01 \%$ over the temperature range [38]. Indeed one would expect any changes to the physical properties of the components to be dominated by the 
lithiation/delithiation of the electrodes during cycling [26] rather than any thermal effects in normal operation.

Further interrogating the initial decrease in the acoustic ToF signal during the charging and discharging processes, it is seen that this is a non-linear process, which can be more clearly identified by examining the individual spectra, as shown in Figure 6.
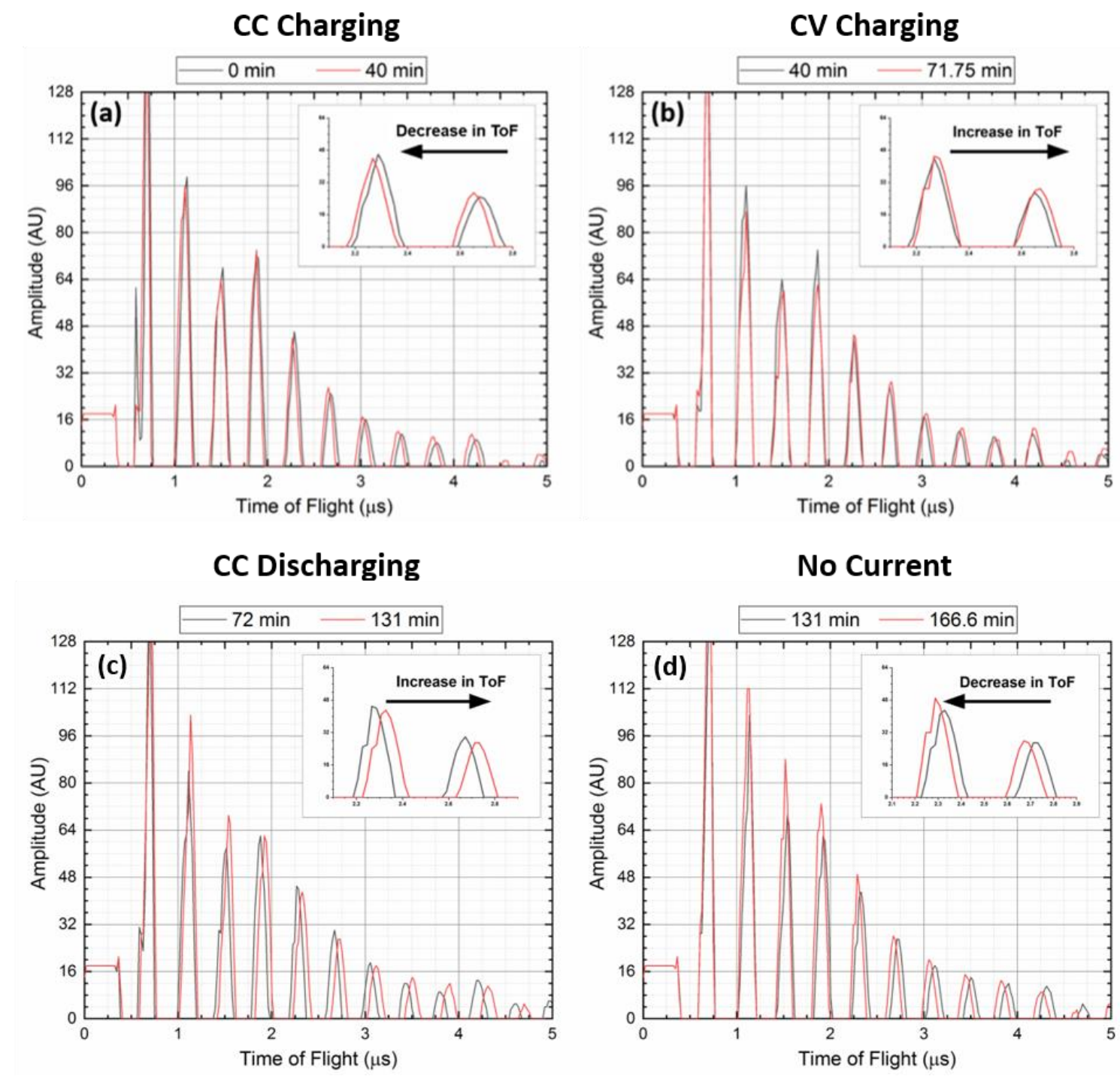

Figure 6 Individual acoustic ToF spectra taken at (a) $0.00 \mathrm{~min}$ (initial SoC), $40.00 \mathrm{~min}$ (end of constant current charging) and (b) $\mathbf{4 0 . 0 0}$ min and $71.75 \mathrm{~min}$ (end of constant voltage charging) showing contrasting behaviour in the acoustic ToF signal resulting in an overall decrease associated with charging. The constant current discharging behaviour can be seen in (c) with the relaxation observed after the discharging was completed shown in $(d)$.

The competing processes shown in Figure 6(a,b) highlight a decrease in the acoustic ToF signal during the constant current charging phase, consistent with an expansion in the electrode 
and/or increase in velocity through the electrode layer, as is expected for both the graphite anode and cathode in a LCO-based cell during charging $[29,30]$. The similarity in the spectra shown in Figure 5 suggests that temperature effects are not a cause of these changes. The increased spacing between the peaks progressing along the spectra, which highlights this behaviour, most clearly is as a result of an increased acoustic ToF for the acoustic signal to penetrate the layers and reflect back to the sensor. The behaviour observed during the constant current charge [seen in Figure 6(a)] is then seen to invert during the constant voltage charging phase [Figure 6(b)]. While this may be interpreted as a reduction in velocity or contraction in the electrode, it is more likely due to a reduction in 'electrochemical stiffness' as the electrodes tend to the open circuit potential. Electrochemical stiffness, which is a measure of the electrochemically induced change in Young's modulus, has been previously reported to be most prevalent at high-rate operation [39]. This phenomena results in the generation of stresses as a result of lithiation and delithiation of electrodes which has been reported to scale proportionally with the rate of operation [39]. In this instance the local heterogeneities in current density, which induce this stress, will tend to subside as the current, which causes electrochemical stiffness, is reduced. An increase in stiffness of the material will also induce electrochemical strains within the system, which have been shown to be caused by the local SoC rather than the lithiation/delithiation process, may result in degradation of the electrode microstructure [39].

The magnitude of the increase in acoustic ToF observed due to the discharge process can be seen to be significantly larger than that observed in Figure $6(a, b)$. The large deviation in the response suggests that a simple, direct, correlation between the acoustic ToF signal and the local SoC does not provide an accurate representation of the condition of the cell. Once again, the results shown in Figure 5 suggest that temperature is not a significant factor in these changes in acoustic ToF. Furthermore, the contrasting behaviour in Figure 6(b) and Figure 6(d), which analyse periods in which the cell is observed to cool, provide further evidence that the changes are induced by alternative processes. Once more, it is suggested the deviations observed in Figure 6(c, d) indicate the presence of an electrochemical stiffness resulting in apparent stresses within the electrode layers. These stresses must manifest themselves through either an apparent increase in density, or more likely through a change in the elasticity of the component materials in the electrode. Further evidence for this is provided in Figure 6(d), which shows the variation in the acoustic ToF signal while the cell is allowed to rest at open circuit potential. A clear 
relaxation in the signal is observed in a similar manner observed to that in Figure 6(b). The fact that the peak locations do not return to their initial location highlights the potential for acoustic ToF analysis to identify SoC; however, these must be performed either at a rested open circuit potential or as a comparative metric against the cell in similar conditions (for example during a known cycle). The difference between the initial and final peak locations may also provide an opportunity to quantify electrochemically induced stiffness in the electrodes of batteries.

Variations in the amplitude of the signal are visible in Figure 4 throughout the cycling of the cell. A general trend of a reduction of the amplitude during the charge cycle is observed, with an increase observed during discharge. These variations are observed to occur at different acoustic ToFs; suggesting that, in this case, the characteristic responses from the anode and cathode are contained within the same peak. This indicates that the resolution of this measurement (20.2 ns) is not sufficient to discretise the acoustic ToF of the respective electrode layers fully. The divergent behaviour of these layers is identifiable by plotting the timeresolved behaviour of a single peak as shown in Figure 7(a), which is composed of six individual spectra, as shown in Figure 7(b).
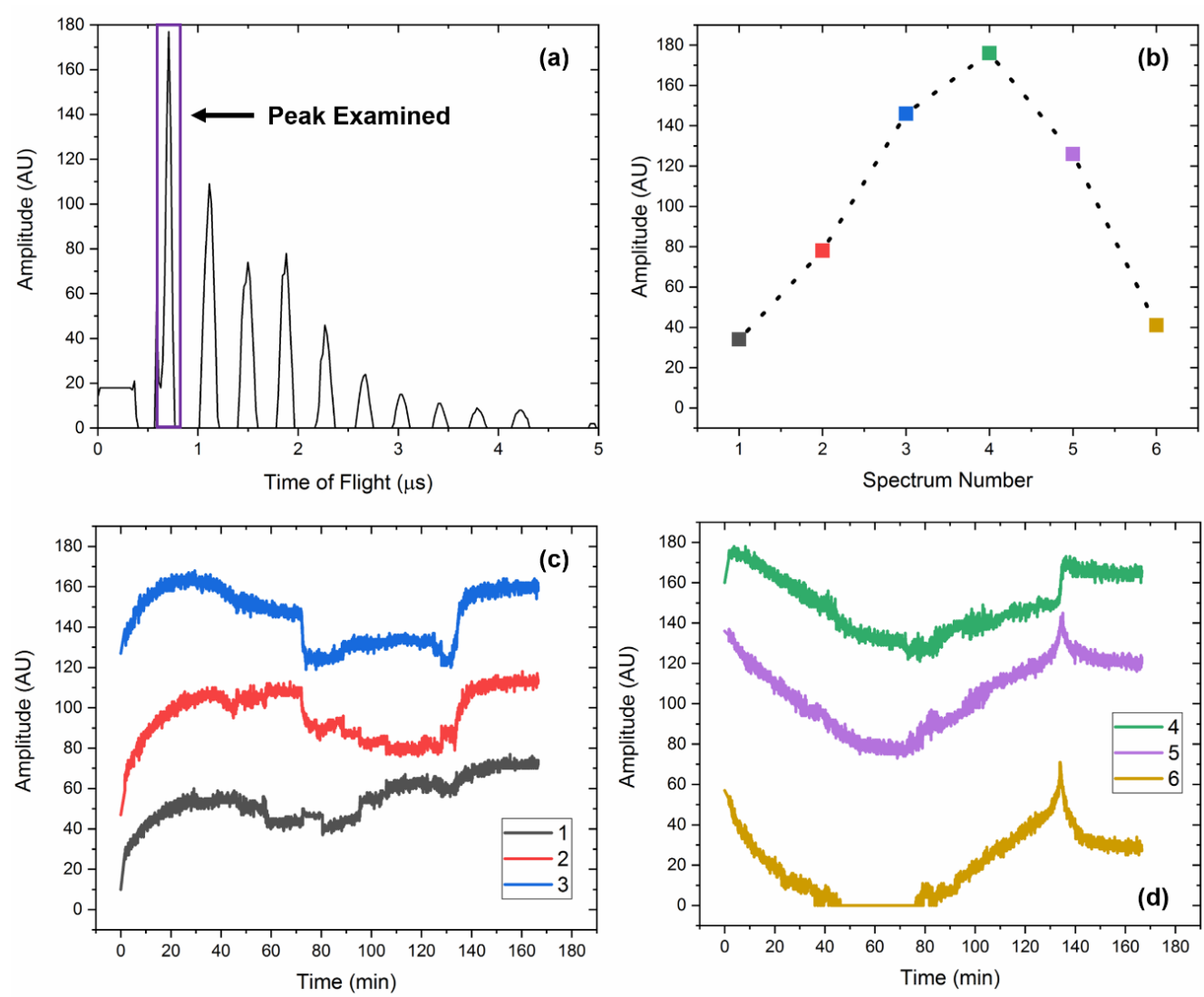

Figure 7 Examining the temporally resolved amplitude response contained within a single peak. The peak investigated is highlighted in (a) with the component measurement locations shown in (b). The divergent 
behaviour in the (c) first three spectra and (d) final three spectra which comprise a single response peak are highlighted with the data collected over an acoustic ToF of $121.45 \mathrm{~ns}$.

It can be clearly seen that each peak throughout the acoustic ToF response signal (b) contains information pertaining to both electrodes, as evidenced by the differing behaviour in Figure 7(c, d). The initial three spectra [shown in Figure 7(b)], are seen to initially increase in amplitude. As highlighted in Equation 4, the acoustic impedance contains contributions from both the density and Young's modulus of the material, it does not, however, depend upon the velocity of propagation of the acoustic waves. As a result, the identification of the component materials is somewhat easier when considering the amplitude. In this instance, the product of the density and Young's modulus is shown to change in a divergent manner during the initial constant current charging with the response in Figure 7(c) increasing and that of Figure 7(d) decreasing. Previous analysis suggested that in a cell similar to that used in this work, the density of the LCO cathode decreases with increasing SoC with the anode behaving in the opposite manner [26]. As such, the response shown in Figure 7(d) can be ascribed to the cathode, with that seen in Figure 7(c) pertaining to the anode. The initial signal in Figure 7(d) is seen to behave in a similar manner as those, which follow until approximately $135 \mathrm{~min}$, potentially due to a movement of the electrode during the $1 \mathrm{C}$ discharge. This suggests that this acoustic ToF response is a 'hybrid' signal providing information for both the anode and cathode,

The effect of the electrochemical stiffness previously observed in the acoustic ToF measurements is very clear in the results outlined in Figure 7. The signals shown in Figure 7(c) indicate a substantial increase in amplitude, and a corresponding reduction in acoustic impedance after the cell reaches open circuit (ca. 137 mins). As the cell is not under any load and is at thermal equilibrium, it is not expected that the density of the electrode will change; therefore, this change in acoustic impedance can only be as a result of a change in the Young's modulus. This reduction suggests that once the load is removed from the cell, the elasticity of the anode increases. The increase in elasticity may arise due to the reduction in the lithiation state of the electrode, which, in turn, reduces the mass of the anode, enabling the relaxation of the graphite. In contrast, the acoustic amplitude of the cathode material is seen to drop significantly, implying a decrease in elasticity. These results suggest that the magnitude of the 
Young's moduli of battery materials are a function not only of local state-of-charge but also of the local applied current at a given time.

The effect of variations in the operating rate was also examined, with the results shown in Figure 8. Once again, the plots shown in Figure 8(b, c) are temporally resolved. The responses shown for the high-rate discharge cycles display similar trends to those shown previously for the $1 \mathrm{C}$ charge/discharge cycle. An initial decrease in the peak locations of the acoustic ToF response is observed followed by a peak shift away from the sensor during the discharging phase, which begins at approximately 68.50 mins. However, it can be seen that the slope of this response increases significantly with increased C-rate. The increased shift in acoustic ToF observed at higher C-rates corresponds to the higher electrochemical stiffness observed under these conditions, as reported by Tavassol et al. [39]. The relaxation of this electrochemically induced stiffness is also seen once the discharging processes are stopped at 98.17 and 76.48 mins respectively, with the acoustic ToF of the peaks contracting towards the sensor. 

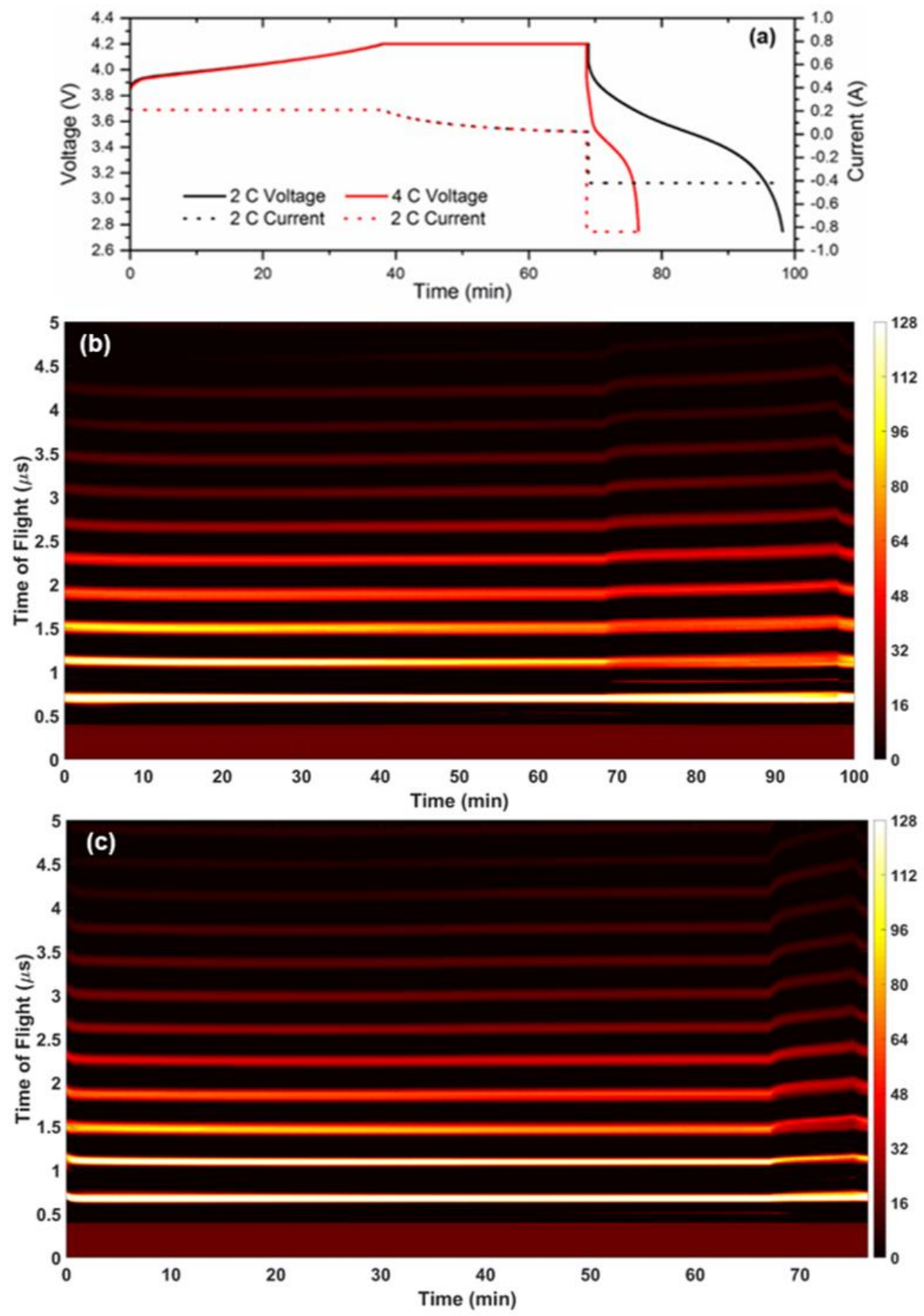

Figure 8 The electrochemical (a) and acoustic signal response for (b) 1C charge, $2 \mathrm{C}$ discharge and (c) 1C charge, 4C discharge cycles. The acoustic ToF spectra (above) shows the acoustic amplitude (measured in AU) and acoustic ToF response of the acoustic measurements during the full discharge cycles and periods during which the cell is allowed to maintain an open circuit potential.

As the discharge of the cell approaches the maximum recommended rate (2 C), some separation in the peaks is distinguishable, as seen in the initial peaks in Figure 8. Two high-amplitude responses are visible towards the later times of discharge in the peak located at approximately 
$1 \mu$ s during the $2 \mathrm{C}$ discharge with the peaks clearly visible during the acoustic ToF relaxation once the discharging has stopped. This separation does, however, manifest itself as a doublet rather than the physical separation of peaks. In contrast, during the $4 \mathrm{C}$ discharge, physical separation of the peaks can be seen at approximately $1.5 \mu \mathrm{s}$. This is likely due to large contractions in the electrodes during extreme discharge, which may indicate a separation or indeed delamination of the electrode layers. The separation of these peaks highlights the potential for the use of acoustic ToF imaging as a diagnostic tool within operational Li-ion systems. By providing the ability to visualise transient physical changes in the cell, which may include delamination due to degradation, or the generation of gas, which provides a layer of high acoustic impedance, the $\mathrm{SoH}$ of the cell can be examined in real time using this technique.

\section{Conclusions}

Temporally resolved ultrasound acoustic ToF analysis was performed on Li-ion pouch cells showing changes in acoustic response during a number of charge-discharge cycles. Electrochemical stiffness, a phenomenon that has been discussed previously in other works, was identified as a key consideration, should the acoustic ToF signal be used to obtain a direct correlation between the physical location, or expansion, of the electrodes. Significant unexpected variations in the acoustic ToF signal were observed during both the charge and discharge phase of the cycle, which indicated the presence of stresses induced by the increase in stiffness. The potential impact of thermal effects was assessed with no change in the acoustic signal observed at the extremes of the batteries temperature. The consistency in acoustic ToF signal obtained during the charging profiles in this work, do, however, indicate that a characteristic response can be obtained for known operating conditions. This characteristic response has the potential to be used as a baseline response to perform comparative operando analysis providing a pathway for the use of this tool in a range of applications, including electric vehicles, which employ prismatic or pouch type cells. Identification of changes in both the anode and cathode (which are contained in the same peak) provides an opportunity to resolve the behaviour of layers at varying depths, enabling approximate locations to be identified for any defects or unusual behaviour.

The variant stresses, which were observed in this work, may manifest themselves in electrodes over time and are likely to induce failures through a range of mechanisms including electrode cracking and delamination. The potential ability, therefore, of acoustic ToF imaging to provide 
a quantification of these stresses may enable further diagnostic capabilities as the battery ages and degrades. Young's moduli of battery materials are a function not only of local SoC but also, of the local applied current at a given time. This finding further highlights the need, and challenge, for an accurate description of the material properties for both electrodes and the identification of any significant current or voltage distributions within the cell. However, the evident relaxation of the electrodes does provide an opportunity to identify the condition of the cell at open circuit, provided that sufficient time is allowed to elapse after any charge or discharge process.

The technique described here has the potential to be deployed at relatively low economic cost and could be integrated into the battery management system of electric vehicles to provide realtime diagnostics of the battery system. Through optimisation of the sensor, this could be achieved with little additional requirement to both the weight and volume allowing powerful, direct, measurements on the SoH and SoC of cells to be obtained when performed using a characteristic baseline response of the cell. This technique has the potential to directly identify cell degradation and failure and if used as a complementary technique to more common methods could provide improved metrics for monitoring battery systems in a range of high impact areas.

\section{Acknowledgements}

The authors would like to acknowledge the EPSRC for supporting the energy storage work in the Electrochemical Innovation Lab (EP/R020973/1; EP/R023581/1; EP/N032888/1; EP/N001583/1; EP/P009050/1; EP/M009394/1; EP/M014371/1). The authors would also like to acknowledge the Royal Academy of Engineering for funding Robinson and Shearing through ICRF1718\1\34 and CiET1718/59 respectively and the Faraday Institution (EP/R042012/1 and EP/R042063/1). The authors also acknowledge the STFC for supporting Shearing and Brett (ST/K00171X/1). The authors would like to acknowledge Quentin Meyer and Daniel Steingart for their assistance in sourcing the equipment and supplying the basic code used to obtain the data.

\section{References}

[1] B. Nykvist, M. Nilsson, Rapidly falling costs of battery packs for electric vehicles, Nature Climate Change, 5 (2015) 329. 
[2] Y. Ding, Z.P. Cano, A. Yu, J. Lu, Z. Chen, Automotive Li-Ion Batteries: Current Status and Future Perspectives, Electrochemical Energy Reviews, 2 (2019) 1-28.

[3] G. Zubi, R. Dufo-López, M. Carvalho, G. Pasaoglu, The lithium-ion battery: State of the art and future perspectives, Renewable and Sustainable Energy Reviews, 89 (2018) 292-308.

[4] J. Vetter, P. Novák, M.R. Wagner, C. Veit, K.C. Möller, J.O. Besenhard, M. Winter, M. Wohlfahrt-Mehrens, C. Vogler, A. Hammouche, Ageing mechanisms in lithium-ion batteries, Journal of Power Sources, 147 (2005) 269-281.

[5] M. Broussely, P. Biensan, F. Bonhomme, P. Blanchard, S. Herreyre, K. Nechev, R.J. Staniewicz, Main aging mechanisms in Li ion batteries, Journal of Power Sources, 146 (2005) 90-96.

[6] M. Ecker, N. Nieto, S. Käbitz, J. Schmalstieg, H. Blanke, A. Warnecke, D.U. Sauer, Calendar and cycle life study of Li(NiMnCo)O2-based 18650 lithium-ion batteries, Journal of Power Sources, 248 (2014) 839-851.

[7] J. Li, E. Murphy, J. Winnick, P.A. Kohl, Studies on the cycle life of commercial lithium ion batteries during rapid charge-discharge cycling, Journal of Power Sources, 102 (2001) 294301.

[8] C.R. Birkl, M.R. Roberts, E. McTurk, P.G. Bruce, D.A. Howey, Degradation diagnostics for lithium ion cells, Journal of Power Sources, 341 (2017) 373-386.

[9] M. Berecibar, I. Gandiaga, I. Villarreal, N. Omar, J. Van Mierlo, P. Van den Bossche, Critical review of state of health estimation methods of Li-ion batteries for real applications, Renewable and Sustainable Energy Reviews, 56 (2016) 572-587.

[10] M.A. Hannan, M.S.H. Lipu, A. Hussain, A. Mohamed, A review of lithium-ion battery state of charge estimation and management system in electric vehicle applications: Challenges and recommendations, Renewable and Sustainable Energy Reviews, 78 (2017) 834-854.

[11] E. Chemali, P.J. Kollmeyer, M. Preindl, A. Emadi, State-of-charge estimation of Li-ion batteries using deep neural networks: A machine learning approach, Journal of Power Sources, 400 (2018) 242-255.

[12] G. Yang, J. Li, Z. Fu, L. Guo, Adaptive state of charge estimation of Lithium-ion battery based on battery capacity degradation model, Energy Procedia, 152 (2018) 514-519.

[13] F. Sun, X. Hu, Y. Zou, S. Li, Adaptive unscented Kalman filtering for state of charge estimation of a lithium-ion battery for electric vehicles, Energy, 36 (2011) 3531-3540.

[14] Y. Xing, W. He, M. Pecht, K.L. Tsui, State of charge estimation of lithium-ion batteries using the open-circuit voltage at various ambient temperatures, Applied Energy, 113 (2014) 106-115.

[15] Y. Zou, X. Hu, H. Ma, S.E. Li, Combined State of Charge and State of Health estimation over lithium-ion battery cell cycle lifespan for electric vehicles, Journal of Power Sources, 273 (2015) 793-803.

[16] K.S. Ng, C.-S. Moo, Y.-P. Chen, Y.-C. Hsieh, Enhanced coulomb counting method for estimating state-of-charge and state-of-health of lithium-ion batteries, Applied Energy, 86 (2009) 1506-1511.

[17] P. Pietsch, V. Wood, X-Ray Tomography for Lithium Ion Battery Research: A Practical Guide, Annual Review of Materials Research, 47 (2017) 451-479. 
[18] M.D.R.R. Kok, J.B.; Weaving, J.S.; Jnawali, A.; Brett, D.J.L.; Shearing, P.R.; Virtual unrolling of spirally-wound lithium-ion cells for correlative degradation studies and predictive fault detection, Sustainable Energy \& Fuels, In Submission (2019).

[19] T. Shibagaki, Y. Merla, G.J. Offer, Tracking degradation in lithium iron phosphate batteries using differential thermal voltammetry, Journal of Power Sources, 374 (2018) 188195.

[20] J.B. Robinson, E. Engebretsen, D.P. Finegan, J. Darr, G. Hinds, P.R. Shearing, D.J.L. Brett, Detection of Internal Defects in Lithium-Ion Batteries Using Lock-in Thermography, ECS Electrochemistry Letters, 4 (2015) A106-A109.

[21] J.B. Robinson, J.A. Darr, D.S. Eastwood, G. Hinds, P.D. Lee, P.R. Shearing, O.O. Taiwo, B. D.J.L, Non-uniform temperature distribution in Li-ion batteries during discharge - A combined thermal imaging, X-ray micro-tomography and electrochemical impedance approach, Journal of Power Sources, 252 (2014) 51-57.

[22] Y. Merla, B. Wu, V. Yufit, N.P. Brandon, R.F. Martinez-Botas, G.J. Offer, Novel application of differential thermal voltammetry as an in-depth state-of-health diagnosis method for lithium-ion batteries, Journal of Power Sources, 307 (2016) 308-319.

[23] G. Davies, K.W. Knehr, B. Van Tassell, T. Hodson, S. Biswas, A.G. Hsieh, D.A. Steingart, State of Charge and State of Health Estimation Using Electrochemical Acoustic Time of Flight Analysis, Journal of The Electrochemical Society, 164 (2017) A2746-A2755.

[24] A.G. Hsieh, S. Bhadra, B.J. Hertzberg, P.J. Gjeltema, A. Goy, J.W. Fleischer, D.A. Steingart, Electrochemical-acoustic time of flight: in operando correlation of physical dynamics with battery charge and health, Energy \& Environmental Science, 8 (2015) 15691577 .

[25] S. Bhadra, A.G. Hsieh, M.J. Wang, B.J. Hertzberg, D.A. Steingart, Anode Characterization in Zinc-Manganese Dioxide AA Alkaline Batteries Using ElectrochemicalAcoustic Time-of-Flight Analysis, Journal of The Electrochemical Society, 163 (2016) A1050A1056.

[26] J.B. Robinson, M. Maier, G. Alster, T. Compton, D.J.L. Brett, P.R. Shearing, Spatially resolved ultrasound diagnostics of Li-ion battery electrodes, Physical Chemistry Chemical Physics, (2019).

[27] P. Ladpli, F. Kopsaftopoulos, F.-K. Chang, Estimating state of charge and health of lithium-ion batteries with guided waves using built-in piezoelectric sensors/actuators, Journal of Power Sources, 384 (2018) 342-354.

[28] D.A. Steingart, https://github.com/dansteingart/nodeforwarder, in, vol. 2018, github.com, 2018.

[29] Y. Koyama, T.E. Chin, U. Rhyner, R.K. Holman, S.R. Hall, Y.M. Chiang, Harnessing the Actuation Potential of Solid-State Intercalation Compounds, Advanced Functional Materials, 16 (2006) 492-498.

[30] X. Wang, Y. Sone, G. Segami, H. Naito, C. Yamada, K. Kibe, Understanding Volume Change in Lithium-Ion Cells during Charging and Discharging Using In Situ Measurements, Journal of The Electrochemical Society, 154 (2007) A14-A21. 
[31] P.R. Shearing, L.E. Howard, P.S. Jørgensen, N.P. Brandon, S.J. Harris, Characterization of the 3-dimensional microstructure of a graphite negative electrode from a Li-ion battery, Electrochemistry Communications, 12 (2010) 374-377.

[32] J. Liu, M. Kunz, K. Chen, N. Tamura, T.J. Richardson, Visualization of Charge Distribution in a Lithium Battery Electrode, The Journal of Physical Chemistry Letters, 1 (2010) 2120-2123.

[33] J.B. Nelson, D.P. Riley, The thermal expansion of graphite from 15 c. to 800 c.: part I. Experimental, Proceedings of the Physical Society, 57 (1945) 477-486.

[34] D.K.L. Tsang, B.J. Marsden, S.L. Fok, G. Hall, Graphite thermal expansion relationship for different temperature ranges, Carbon, 43 (2005) 2902-2906.

[35] E.J. Cheng, N.J. Taylor, J. Wolfenstine, J. Sakamoto, Elastic properties of lithium cobalt oxide (LiCoO2), Journal of Asian Ceramic Societies, 5 (2017) 113-117.

[36] K. Wang, R.R. Reeber, The perfect crystal, thermal vacancies and the thermal expansion coefficient of aluminium, Philosophical Magazine A, 80 (2000) 1629-1643.

[37] P. Bing, X. Hui-min, H. Tao, A. Asundi, Measurement of coefficient of thermal expansion of films using digital image correlation method, Polymer Testing, 28 (2009) 75-83.

[38] D.M.S. McEligot, W.D.; Cottle, D.L.; Valentin, F.I., Thermal Properties of G-348 Graphite, in, Idaho National Laboratory, 2016.

[39] H. Tavassol, E.M.C. Jones, N.R. Sottos, A.A. Gewirth, Electrochemical stiffness in lithium-ion batteries, Nature Materials, 15 (2016) 1182. 